\title{
Fresnel coefficients for a phase conjugator
}

\author{
Henk F. Arnoldus
}

Department of Physics, Mendel Hall, Villanova University, Villanova, Pennsylvania 19085

Thomas F. George

Department of Physics, 239 Fronczak Hall, State University of New York at Buffalo, Buffalo, New York 14260

\author{
Received October 27, 1987; accepted September 28, 1988
}

\begin{abstract}
Optical phase conjugation by four-wave mixing is examined in detail. The Fresnel coefficients for reflection and transmission of a plane wave irradiating the surface of the phase conjugator are calculated. It appears that for normal incidence and a weak nonlinear interaction the device produces the phase-conjugated beam with respect to the incoming beam. For finite angles of incidence or stronger nonlinearities in the crystal, the generated wave deviates from the ideal conjugated wave, and also a second, specularly reflected, wave appears.
\end{abstract}

\section{INTRODUCTION}

After the first demonstrations ${ }^{1-4}$ of the feasibility of generating phase-conjugated electromagnetic waves (with respect to a reference wave), this technique has found important applications in optical engineering, especially in the design of devices for the production of high-quality laser beams. If a light ray is reflected by a phase-conjugating mirror, then its wave front is reversed. This implies that a diverging beam emanates as converging rather than as diverging, which would be the situation for an ordinary mirror. In this fashion, a distorted wave front can be corrected, after reflection by a phase conjugator (PC), by letting it pass through the same device that built up the distortion. ${ }^{5-7}$

Since wave-front reversal by PC's appears to work so well, one can now anticipate more sophisticated applications. In particular, lifetime modifications of atoms, which are due to the fact that the atom radiates its fluorescence (in spontaneous decay) in the vicinity of a PC, are expected to be dramatic. ${ }^{8}$ Emitted dipole radiation diverges from its source, and a subsequent reflection by a PC can focus the wave exactly back onto the atom. Stimulated absorption of photons can then conceivably lead to (effective) infinite lifetimes of excited atomic states. This in turn leads to a reduction of the natural linewidth, which might have consequences for frequency standards. It was predicted ${ }^{9}$ that the linewidth would vanish identically, if the reflectivity of the mirror equaled unity, and this property would be independent of the distance between the atom and the PC.

Although wave-front reversal has been proved to be possible in general, it is more difficult to achieve in practice if one wants to extend the horizon of its applications. Let us represent the electric field by its Fourier integral

$$
\mathbf{E}(\mathbf{r}, t)=\frac{1}{2 \pi} \int_{-\infty}^{\infty} \mathrm{d} \omega e^{-i \omega t} \hat{\mathbf{E}}(\mathbf{r}, \omega) .
$$

From $\mathbf{E}(\mathbf{r}, t)^{*}=\mathbf{E}(\mathbf{r}, t)$ we obtain the relation

$$
\hat{\mathbf{E}}(\mathbf{r}, \omega)^{*}=\hat{\mathbf{E}}(\mathbf{r},-\omega) \text {. }
$$

On the other hand, the phase-conjugated replica of $\mathbf{E}(\mathbf{r}, t)$ follows from the substitution $\hat{\mathbf{E}}(\mathbf{r}, \omega) \rightarrow \hat{\mathbf{E}}(\mathbf{r}, \omega)^{*}$ in Eq. (1.1), and combination with Eq. (1.2) then yields

$$
\frac{1}{2 \pi} \int_{-\infty}^{\infty} \mathrm{d} \omega e^{-i \omega t} \hat{\mathbf{E}}(\mathbf{r}, \omega)^{*}=\mathbf{E}(\mathbf{r},-t) .
$$

Hence perfect phase conjugation is identical to time reversal, and it is easy to argue that it is impossible to construct a device that can accomplish that.

Time reversal (looking into the future) violates causality. For the example of fluorescence, this would imply that at the time the photon is emitted the atom already knows that the phase-conjugated wave will be reflected back. If the distance between atom and mirror, divided by the speed of light, is much larger than the atomic lifetime, then the presence of the PC should not affect the optical properties of the atom any more, according to the principles of special relativity. Therefore more profound understanding of phase conjugation requires a time (or frequency) resolution in the description, and in such a way that causality is preserved. Besides that, the literature ${ }^{10-18}$ on reflection by PC's is largely restricted to the case of normal incidence of the probe field. Dipole radiation, for instance, is a spherical wave, and consequently it is imperative to take a nonzero parallel component of the incident wave vector into consideration.

\section{CONSTITUTIVE EQUATION}

If radiation is scattered by a vacuum-material interface, then the reflected field often acquires a phase-conjugated component. ${ }^{19}$ Since we are interested in the basic possibilities and limitations of producing phase-conjugated radiation, we consider the simplest configuration, which is experimentally realizable $e^{20}$ and has all the desired features. As the active medium we choose a crystal that is transparent (unit dielectric constant) for the frequency range under consideration but has a significant third-order susceptibility $\chi^{(3)}(\omega)$. Two strong counterpropagating and linearly polarized laser beams with frequency $\bar{\omega}>0$ are assumed to excite the nonlinear interaction. A relatively weak incident (probe) wave then induces a polarization $\mathbf{P}(\mathbf{r}, t)$ in the crys- 
tal. Corresponding oscillating charges then emit radiation, which gives rise to a reflected, phase-conjugated wave. From the theory of four-wave mixing ${ }^{21,22}$ we know that the (Fourier transform of the) polarization is then related to the electric field by

$$
\hat{\mathbf{P}}(\mathbf{r}, \omega)=\epsilon_{0} \hat{f}(\bar{\omega}-\omega) * \hat{\mathbf{E}}(\mathbf{r}, \omega-2 \bar{\omega}), \quad \omega>0 .
$$

Here, $\mathbf{E}$ represent the electric field at $\mathbf{r}$ in the crystal, but it does not include the two pump fields. The function $\hat{f}(\omega)$ represents the nonlinear material and will remain unspecified further. This quantity has the same status as the frequency-dependent first-order susceptibility $\chi^{(1)}(\omega)$ for dielectrics. In the present situation, the $\omega$ dependence of $\hat{f}$ equals the $\omega$ dependence of $\chi^{(3)}$, shifted over the setting frequency $\vec{\omega}$ of the phase conjugator. Furthermore, $\hat{f}$ is proportional to the products of the amplitudes of the two pump beams.

From $\mathbf{P}(\mathbf{r}, t)^{*}=\mathbf{P}(\mathbf{r}, t)$ we find that $\hat{\mathbf{P}}(\mathbf{r}, \omega)^{*}=\hat{\mathbf{P}}(\mathbf{r},-\omega)$, and with Eq. (1.2) we then obtain

$$
\hat{\mathbf{P}}(\mathbf{r}, \omega)=\epsilon_{0} \hat{f}(\bar{\omega}+\omega) \hat{\mathbf{E}}(\mathbf{r}, 2 \bar{\omega}+\omega), \quad \omega<0,
$$

the polarization for negative frequencies. Equations (2.1) and (2.2) give $\hat{\mathbf{P}}(\mathbf{r}, \omega)$ for every $\omega$, and together they will be considered the constitutive relation for a $\mathrm{PC}$.

Because the frequency dependence of $\hat{f}(\omega)$ represents the frequency dependence of $\chi^{(3)}(\omega)$ around the setting $\bar{\omega}$, the function $\hat{f}(\omega)$ should be strongly peaked around $\omega \sim 0$. From Eq. (2.2) we then see that $\hat{\mathbf{P}}(\mathbf{r}, \omega)$ can be nonzero only for frequencies $\omega \sim-\bar{\omega}$. Its value is proportional to $\hat{\mathbf{E}}$, evaluated at the frequency $2 \bar{\omega}+\omega \sim \bar{\omega}$. In other words, Eq. (2.2) expresses that $\hat{\mathbf{P}}$ around $-\bar{\omega}$ is determined by $\hat{\mathbf{E}}$ around $\bar{\omega}$ and is zero if $\omega$ is sufficiently far away from $-\bar{\omega}$. With this in mind, the Fourier inverse of Eq. (2.2) is readily found to be

$$
\mathbf{P}^{(-)}(\mathbf{r}, t)=\epsilon_{0} e^{i \bar{\omega} t} \int_{0}^{\infty} \mathrm{d} \tau f(\tau) \exp [i \bar{\omega}(t-\tau)] \mathbf{E}^{(+)}(\mathbf{r}, t-\tau)
$$

where $(-)$ and $(+)$ indicate the negative- and positive-frequency parts, respectively. The function $f(\tau)$ is related to $\hat{f}(\omega)$ by

$$
\hat{f}(\omega)=\int_{0}^{\infty} \mathrm{d} \tau e^{i \omega \tau} f(\tau)
$$

which is a Fourier integral if we set $f(\tau<0)=0$. Equation (2.3) expresses that the polarization at time $t$ is determined by the electric field in the past only. Therefore the mechanism of phase conjugation is causal.

\section{COUPLED-WAVE EQUATIONS}

In terms of a polarization, the Fourier-transformed Maxwell equations read as

$$
\begin{gathered}
\nabla \cdot\left[\epsilon_{0} \hat{\mathbf{E}}(\mathbf{r}, \omega)+\hat{\mathbf{P}}(\mathbf{r}, \omega)\right]=0, \\
\nabla \cdot \hat{\mathbf{B}}(\mathbf{r}, \omega)=0, \\
\nabla \times \hat{\mathbf{E}}(\mathbf{r}, \omega)-i \omega \hat{\mathbf{B}}(\mathbf{r}, \omega)=0, \\
\mu_{0}^{-1} \nabla \times \hat{\mathbf{B}}(\mathbf{r}, \omega)+i \omega\left[\epsilon_{0} \hat{\mathbf{E}}(\mathbf{r}, \omega)+\hat{\mathbf{P}}(\mathbf{r}, \omega)\right]=0,
\end{gathered}
$$

which should be obeyed for every $\omega$ separately. Outside the $\mathrm{PC}$ we have $\hat{\mathbf{P}}=0$; inside we set $\hat{\mathbf{P}}$ equal to expression (2.1) or
(2.2), depending on the sign of $\omega$. Furthermore, Eqs. (3.1)(3.4) imply the boundary conditions at the interface in the usual way.

The polarization $\hat{\mathbf{P}}$ at frequency $\omega$ is expressed in $\hat{\mathbf{E}}$ at a different frequency, and therefore Eqs. (3.1) and (3.4) couple sets of Maxwell equations for different frequencies. If we take a fixed frequency $\omega_{1} \sim \bar{\omega}$, then the polarization $\hat{\mathbf{P}}\left(\mathbf{r}, \omega_{1}\right)$ is brought about by the electric field at $\omega_{1}-2 \bar{\omega}<0$, according to Eq. (2.1). On the other hand, the polarization at $\omega_{1}-$ $2 \bar{\omega}$ is induced by $\hat{\mathbf{E}}\left(\mathbf{r}, \omega_{1}\right)$, as follows from Eq. (2.2). Therefore Maxwell's equations couple positive and negative frequencies two by two. If we take $\omega_{1} \sim \bar{\omega}>0$, then this frequency couples with $\omega_{2} \sim-\bar{\omega}<0$, and their relation is

$$
\omega_{1}-\bar{\omega}=\omega_{2}+\bar{\omega}
$$

For the fields inside the PC we eliminate $\hat{\mathbf{B}}$ with Eq. (3.3):

$$
\hat{\mathbf{B}}(\mathbf{r}, \omega)=-i \omega^{-1} \nabla \times \hat{\mathbf{E}}(\mathbf{r}, \omega) \quad \text { for } \omega=\omega_{1}, \omega_{2} .
$$

Then Eq. (3.2) is automatically satisfied, and Maxwell's equations are equivalent to

$$
\begin{gathered}
\nabla \cdot \hat{\mathbf{E}}(\mathbf{r}, \omega)=0 \quad \text { for } \omega=\omega_{1}, \omega_{2} \\
\nabla^{2} \hat{\mathbf{E}}\left(\mathbf{r}, \omega_{1}\right)+\left(\omega_{1} / c\right)^{2}\left[\hat{\mathbf{E}}\left(\mathbf{r}, \omega_{1}\right)+\hat{f}\left(\bar{\omega}-\omega_{1}\right) * \hat{\mathbf{E}}\left(\mathbf{r}, \omega_{2}\right)\right]=0 \\
\nabla^{2} \hat{\mathbf{E}}\left(\mathbf{r}, \omega_{2}\right)+\left(\omega_{2} / c\right)^{2}\left[\hat{\mathbf{E}}\left(\mathbf{r}, \omega_{2}\right)+\hat{f}\left(\bar{\omega}+\omega_{2}\right) \hat{\mathbf{E}}\left(\mathbf{r}, \omega_{1}\right)\right]=0
\end{gathered}
$$

Equation (3.7) expresses that the electric field is transverse, and Eqs. (3.8) and (3.9) show that the fields at $\omega_{1}$ and $\omega_{2}$ obey a set of coupled-wave equations.

\section{PLANE WAVES}

Solutions of the set Eqs. (3.7)-(3.9) are easily found. If we try

$$
\begin{gathered}
\hat{\mathbf{E}}_{a}\left(\mathbf{r}, \omega_{1}\right)=\mathbf{E}_{a} \exp \left(i \mathbf{k}_{a} \cdot \mathbf{r}\right), \\
\hat{\mathbf{E}}_{a}\left(\mathbf{r}, \omega_{2}\right)=\eta_{a} \mathbf{E}_{a} \exp \left(i \mathbf{k}_{a} \cdot \mathbf{r}\right),
\end{gathered}
$$

then Eqs. (3.7)-(3.9) become

$$
\begin{gathered}
\mathbf{k}_{a} \cdot \hat{\mathbf{E}}_{a}=0, \\
k_{a}{ }^{2}=\left(\omega_{1} / c\right)^{2}\left[1+\eta_{a} \hat{f}\left(\bar{\omega}-\omega_{1}\right)^{*}\right], \\
k_{a}{ }^{2}=\left(\omega_{2} / c\right)^{2}\left[1+\eta_{a}{ }^{-1} \hat{f}\left(\bar{\omega}+\omega_{2}\right)\right] .
\end{gathered}
$$

Equations (4.4) and (4.5) both express the (complex-valued) wave number in the ratio of amplitudes $\eta_{a}$ of the $\omega_{1}$ and $\omega_{2}$ waves. Equating the right-hand sides gives a quadratic equation for $\eta_{a}$, which admits of two acceptable solutions. For reasons that will become clear in what follows, we choose

$$
\eta_{a}=\frac{\left[1-\left(\omega_{1} / \omega_{2}\right)^{2}\right](1-D)}{2\left(\omega_{1} / \omega_{2}\right)^{2} \hat{f}\left(\bar{\omega}-\omega_{1}\right)^{*}}
$$

in terms of the parameter

$$
D=\left\{1+\frac{4\left(\omega_{1} / \omega_{2}\right)^{2} \hat{f}\left(\bar{\omega}-\omega_{1}\right)^{*} f\left(\bar{\omega}+\omega_{2}\right)}{\left[1-\left(\omega_{1} / \omega_{2}\right)^{2}\right]^{2}}\right\}^{1 / 2} .
$$

Equations (4.6) and (4.7) express $\eta_{a}$ in the given function $\hat{f}$, 
and with Eq. (4.4) we find $k_{a}{ }^{2}$. For a given frequency dependence of $\hat{f}(\omega)$, Eq. (4.4) is then the dispersion relation for the PC.

Instead of retaining two possible values for $\eta_{a}$, we seek a second solution of the form

$$
\begin{gathered}
\hat{\mathbf{E}}_{b}\left(\mathbf{r}, \omega_{1}\right)=\eta_{b} \mathbf{E}_{b} \exp \left(i \mathbf{k}_{b} \cdot \mathbf{r}\right), \\
\hat{\mathbf{E}}_{b}\left(\mathbf{r}, \omega_{2}\right)=\mathbf{E}_{b} \exp \left(i \mathbf{k}_{b} \cdot \mathbf{r}\right),
\end{gathered}
$$

and in the same way we find

$$
\begin{gathered}
\mathbf{k}_{b} \cdot \mathbf{E}_{b}=0, \\
k_{b}^{2}=\left(\omega_{2} / c\right)^{2}\left[1+\eta_{b} \hat{f}\left(\bar{\omega}+\omega_{2}\right)\right], \\
k_{b}^{2}=\left(\omega_{1} / c\right)^{2}\left[1+\eta_{b}{ }^{-1} \hat{f}\left(\bar{\omega}-\omega_{1}\right)^{*}\right] .
\end{gathered}
$$

Again, there are two solutions for $\eta_{b}$, but now we take

$$
\eta_{b}=\frac{\left[\left(\omega_{1} / \omega_{2}\right)^{2}-1\right](1-D)}{2 \hat{f}\left(\bar{\omega}+\omega_{2}\right)} .
$$

Substitution of $\eta_{b}$ into Eq. (4.11) then gives a second branch of the dispersion relation. It is easy to check that the $b$ solution is precisely the discarded $a$ solution.

In the limit $\vec{f} \rightarrow 0$, corresponding to a switch-off of the interaction, we find that

$$
\eta_{a} \rightarrow 0, \quad k_{a}^{2} \rightarrow\left(\omega_{1} / c\right)^{2},
$$

and hence the $\omega_{2}$ wave disappears. The dispersion relation for the $\omega_{1}$ wave is the same as for a wave in a vacuum. This implies that the $a$ solution is essentially an $\omega_{1}$ wave, but owing to the nonlinear interaction $\hat{f} \neq 0$ there is a mixing with the $\omega_{2}$ wave, which is excited with a relative amplitude $\eta_{a}$. The second, suppressed solution for $\eta_{a}$ would give $\eta_{a} \rightarrow$ $\infty$ for $\vec{f} \rightarrow 0$. Since the amplitude of the $\omega_{2}$ wave must remain finite, this would imply that $\mathbf{E}_{a} \rightarrow 0, \eta_{a} \mathbf{E}_{a}$ finite, indicating that the $\omega_{1}$ wave disappears in comparison with the $\omega_{2}$ wave. The property $\eta_{a} \rightarrow \infty$ for $\hat{f} \rightarrow 0$ is inconvenient. Therefore we have introduced the second solution with the normalization as given by Eqs. (4.8) and (4.9). For $\hat{f} \rightarrow 0$ we now obtain

$$
\eta_{b} \rightarrow 0, \quad k_{b}^{2} \rightarrow\left(\omega_{2} / c\right)^{2},
$$

showing that the $b$ solution is essentially an $\omega_{2}$ wave, modified by the nonlinear interaction.

From Eqs. (4.1) and (4.2) we notice that the $\omega_{1}$ and $\omega_{2}$ waves seem to have the same wave vector and polarization and that their ratio of amplitudes equals $\eta_{a}$. However, because $\omega_{1}>0$ and $\omega_{2}<0$, the waves are counterpropagating. In the time domain, the $\omega_{2}$ wave has a factor $\exp \left(i \mathbf{k}_{a} \cdot \mathbf{r}-\right.$ $\left.i \omega_{2} t\right)+$ c.c., which represents a wave with wave vector $-\mathbf{k}_{a}$. Finally, we mention two interesting relations between the $a$ and $b$ solutions. For the amplitude factors we have

$$
\frac{\eta_{a}}{\eta_{b}}=-\left(\frac{\omega_{2}}{\omega_{1}}\right)^{2} \frac{\hat{f}\left(\bar{\omega}+\omega_{2}\right)}{\hat{f}\left(\bar{\omega}-\omega_{1}\right)^{*}}
$$

and the wave numbers are related according to

$$
k_{a}{ }^{2}+k_{b}{ }^{2}=\left(\omega_{1} / c\right)^{2}+\left(\omega_{2} / c\right)^{2},
$$

independent of the interaction strength $\hat{f}$.

\section{WAVES AT AN INTERFACE}

Consider position space to be divided in vacuum $(z>0)$ and a $\mathrm{PC}(z<0)$, separated by a plane boundary $(z=0)$. Incident upon this interface, and from the vacuum side, is a plane monochromatic wave with frequency $\omega_{1}>0$,

$$
\hat{\mathbf{E}}_{i}\left(\mathbf{r}, \omega_{1}\right)=\mathbf{E} \exp (i \mathbf{k} \cdot \mathbf{r}),
$$

with amplitude and polarization $\mathbf{E}$ and wave vector $\mathbf{k}$ assumed to be given. From Maxwell's Eqs. (3.1)-(3.4) we find the constraints

$$
k^{2}=\left(\omega_{1} / c\right)^{2}, \quad \mathbf{k} \cdot \mathbf{E}=0 .
$$

If we write $\mathbf{k}=\mathbf{k}_{\|}+\mathbf{k}_{\perp}$, where $\|$ and $\perp$ refer to the plane $z=$ 0 , then the $z$ component of $\mathbf{k}$ is

$$
k_{z}=-\left(k^{2}-k_{\|}^{2}\right)^{1 / 2} .
$$

The question is what the reflected and transmitted waves are. In this section we establish which waves occur and determine their wave vectors. In Section 6 we evaluate their amplitudes (including phase and polarization), e.g., the Fresnel coefficients.

Waves in $z>0$ and in $z<0$ must match at $z=0$ according to the boundary conditions. Every plane wave contains a factor $\exp \left(i \mathbf{k}_{\alpha} \cdot \mathbf{r}\right)$, which equals $\exp \left(i \mathbf{k}_{\alpha, \|} \cdot \mathbf{r}\right)$ at $z=0$. Boundary conditions can be satisfied only if these exponentials cancel, implying that the parallel component of every wave vector must be identical, e.g.,

$$
\mathbf{k}_{\alpha, \|}=\mathbf{k}_{\|}
$$

for any wave vector $\mathbf{k}_{\alpha}$ in $z>0$ and $z<0$. Then, in both $z>0$ and $z<0$, the value of $k_{\alpha}^{2}$ follows from the dispersion relation, given the frequency, which in turn gives for the perpendicular component the two possibilities $k_{\alpha, z}= \pm\left(k_{\alpha}{ }^{2}\right.$ $\left.-k_{\|}^{2}\right)^{1 / 2}$. The sign in front of the square root determines whether the wave travels in the $+z$ or the $-z$ direction. Since the incident wave has frequency $\omega_{1}$, which couples only to $\omega_{2}$, these considerations limit the number of possible plane waves to four in $z>0$ and eight in $z<0$.

Let us first look at the region $z>0$. Besides the incident wave at frequency $\omega_{1}$, the only other possibility for waves at $\omega_{1}$ is the common reflected wave with

$$
k_{r, z}=-k_{z}>0 .
$$

Therefore the most general expression for the electric field at frequency $\omega_{1}$ in $z>0$ reads as

$$
\hat{\mathbf{E}}\left(\mathbf{r}, \omega_{1}\right)=\mathbf{E} \exp (i \mathbf{k} \cdot \mathbf{r})+\mathbf{E}_{r} \exp \left(i \mathbf{k}_{r} \cdot \mathbf{r}\right),
$$

with only $\mathbf{E}_{r}$ yet to be determined. For the field at $\omega_{2}$, we again have two possible waves, which differ only in their sign of the $z$ component of the wave vector. Of course, it is tacitly assumed that the incident wave is the only external field, which implies that an $\omega_{2}$ wave in $z>0$ can travel only in the $+z$ direction. We shall refer to this wave as the phaseconjugated wave, and it is represented by

$$
\hat{\mathbf{E}}\left(\mathbf{r}, \omega_{2}\right)=\mathbf{E}_{\mathrm{PC}} \exp \left(i \mathbf{k}_{\mathrm{PC}} \cdot \mathbf{r}\right) .
$$

The fact that a wave with $\omega_{2}<0$ propagates in a direction opposite its wave vector then gives

$$
k_{\mathrm{PC}, z}=-\left[\left(\omega_{2} / c\right)^{2}-k_{\|}{ }^{2}\right]^{1 / 2} .
$$


If the incident field is exactly on resonance with the $\mathrm{PC}$, we then have $\omega_{1}=\bar{\omega}, \omega_{2}=-\bar{\omega}$, and $k_{\mathrm{PC}, z}=k_{z}$. Combining this with Eq. (5.4) shows that in this case $\mathbf{k}_{\mathrm{PC}}=\mathbf{k}$, and hence the $\mathrm{PC}$ wave and the incident wave counterpropagate exactly. Therefore the field at $\omega_{2}$ in $z>0$ is the phase-conjugated replica of the incident field (possibly apart from polarization and amplitude), if $\omega_{1}=\bar{\omega}$. Furthermore, we notice that for $-\omega_{2} \neq \omega_{1}, k_{\mathrm{PC}, z}$ can be imaginary, corresponding to an evanescent wave.

In the PC we have the independent $a$ and $b$ solutions from Section 4. Both solutions can occur with $a+$ or $a-$ in the definition of the $z$ component of the wave vector. This amounts to four different solutions, and each of them consists of two waves (at $\omega_{1}$ and $\omega_{2}$ ). For the corresponding problem with a dielectric, we know that we can discard the wave that propagates in the $+z$ direction, but for a $\mathrm{PC}$ it is not obvious which waves are the causal waves. This results from the fact that every solution consists of two counterpropagating waves. The only clue at this stage is that, in the limit $\hat{f} \rightarrow 0$, the incident field must propagate undisturbed through the crystal, and every other component must vanish. This corresponds to the $a$ solution with

$$
k_{a, z}=-\left(k_{a}^{2}-k_{\|}^{2}\right)^{1 / 2} \text {. }
$$

Whether the other three solutions are excited by the incident field seems to be impossible to find out a priori. We have to consider the full solution for $z<0$, calculate all amplitudes by matching boundary values, and then require that all fields disappear in the limit $\mathbf{E} \rightarrow 0$. In carrying out this procedure, we have found that only the $b$ solution with

$$
k_{b, z}=\left(k_{b}{ }^{2}-k_{\|}{ }^{2}\right)^{1 / 2}
$$

is excited by the incident wave. This field corresponds to an $\omega_{2}$-like wave, which propogates in the $-z$ direction. For the field in $z<0$ we can now write

$$
\begin{aligned}
& \hat{\mathbf{E}}\left(\mathbf{r}, \omega_{1}\right)=\mathbf{E}_{a} \exp \left(i \mathbf{k}_{a} \cdot \mathbf{r}\right)+\eta_{b} \mathbf{E}_{b} \exp \left(i \mathbf{k}_{b} \cdot \mathbf{r}\right), \\
& \hat{\mathbf{E}}\left(\mathbf{r}, \omega_{2}\right)=\eta_{a} \mathbf{E}_{a} \exp \left(i \mathbf{k}_{a} \cdot \mathbf{r}\right)+\mathbf{E}_{b} \exp \left(i \mathbf{k}_{b} \cdot \mathbf{r}\right),
\end{aligned}
$$

and only $\mathbf{E}_{a}$ and $\mathbf{E}_{b}$ remain to be determined.

\section{FRESNEL COEFFICIENTS}

The fields from Section 5 must be matched across $z=0$, with the conditions that $\left(\epsilon_{0} \hat{\mathbf{E}}+\hat{\mathbf{P}}\right)_{\perp}, \hat{\mathbf{E}} \|$, and $\hat{\mathbf{B}}$ be continuous for both $\omega_{1}$ and $\omega_{2}$. Furthermore, we have the restrictions that $\mathbf{k}_{\alpha} \cdot \mathbf{E}_{\alpha}=0$ for every wave. This procedure fixes the amplitudes $\mathbf{E}_{r}, \mathbf{E}_{\mathrm{PC}}, \mathbf{E}_{a}$, and $\mathbf{E}_{b}$ in terms of $\mathbf{E}$. Notice that two components of the field for $z<0$ acquire an additional factor of $\eta_{b}$ or $\eta_{a}$ in their amplitudes, according to Eqs. (5.11) and (5.12).

As usual, it is advantageous to distinguish between an $s$ (三surface) and a $p$ - (三plane) polarized incident wave. Then all other waves are $s$ or $p$ polarized. In Fig. 1 we have summarized the polarization convention that is adopted here. All unit vectors are normalized as $\mathbf{e}_{\alpha} \cdot \mathbf{e}_{\alpha}=1$, and their directions follow from $\mathbf{e}_{\alpha} \cdot \mathbf{k}_{\alpha}=0$ in combination with the convention from Fig. 1 . Since the $\mathbf{k}_{\alpha}$ 's are not necessarily real, the unit vectors for $p$ polarization will be complex, in general. Then the Fresnel coefficients for $s$ waves are defined by the notation
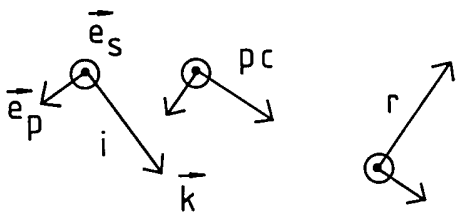

$+7$

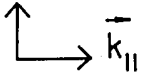

TाIIIIIIIIIIIIIIIIIIIIIIIIIIIIIII
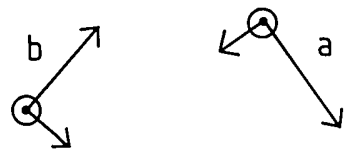

Fig. 1. Polarization convention for the various waves. All $s$-polarization vectors, perpendicular to the plane of incidence, point in the same direction. For $p$-polarized waves we choose the unit vectors such that their $z$ components are negative in the limit $\hat{f} \rightarrow 0$ (for $\hat{f} \neq$ 0 the $z$ components might be complex). The arrows with letters next to them indicate the wave vectors. Their parallel components are all equal to $\mathbf{k}_{\|}$, and the figure shows the sign of their $z$ component in the limit $\hat{f} \rightarrow 0$.

$$
\begin{gathered}
\mathbf{E}=E \mathbf{e}_{s}, \\
\mathbf{E}_{r}=R_{s} E \mathbf{e}_{r, s}, \quad \mathbf{E}_{\mathrm{PC}}=P_{s} E \mathbf{e}_{\mathrm{PC}, s}, \\
\mathbf{E}_{a}=T_{a, s} E \mathbf{e}_{a, s}, \quad \mathbf{E}_{b}=T_{b, s} E \mathbf{e}_{b, s},
\end{gathered}
$$

and for an incident $p$ wave we replace the subscripts $s$ by $p$. We obtain the results that

$$
\begin{gathered}
R_{s}=\frac{1}{Z_{s}}\left[\left(k_{z}-k_{a, z}\right)\left(k_{\mathrm{PC}, z}-k_{b, z}\right)\right. \\
\left.+\eta_{a} \eta_{b}\left(k_{a, z}-k_{\mathrm{PC}, z}\right)\left(k_{z}-k_{b, z}\right)\right], \\
P_{s}=\frac{1}{Z_{s}} 2 \eta_{a} k_{z}\left(k_{a, z}-k_{b, z}\right), \\
T_{a, s}=\frac{1}{Z_{s}} 2 k_{z}\left(k_{\mathrm{PC}, z}-k_{b, z}\right), \\
R_{p}=\frac{1}{Z_{p}}\left[\left(k_{z} \frac{k_{a}{ }^{2}}{k^{2}}-k_{a, z}\right)\left(k_{\mathrm{PC}, z} \frac{k_{b}{ }^{2}}{k_{\mathrm{PC}}{ }^{2}}-k_{b, z}\right)\right. \\
+\eta_{a} \eta_{b}\left(k_{a, z}-k_{\mathrm{PC}, z} \frac{1}{Z_{\mathrm{PC}}{ }^{2}} 2 \eta_{a} k_{z}\left(k_{a, z}-k_{\mathrm{PC}, z}\right),\right. \\
\left.P_{p}=\frac{1}{Z_{p}} 2 \eta_{a} k_{z} \frac{k_{\mathrm{PC}}}{k}\left(k_{a, z} \frac{k_{b}{ }^{2}}{k_{\mathrm{PC}}{ }^{2}}-k_{b, z}\right)\right], \\
T_{a, p}=\frac{1}{Z_{p}} 2 k_{z} \frac{k_{a}}{k}\left(k_{\mathrm{PC}, z} \frac{k_{b}{ }^{2}}{k_{\mathrm{PC}}{ }^{2}}-k_{\mathrm{PC}}{ }^{2}\right), \\
\left.Z_{p}{ }^{2}\right) \\
\eta_{a} k_{z} \frac{k_{b}}{k}\left(k_{a, z}-k_{\mathrm{PC}, z} \frac{k_{a}{ }^{2}}{k_{\mathrm{PC}}{ }^{2}}\right),
\end{gathered}
$$

where we have introduced the abbreviations 


$$
\begin{gathered}
Z_{s}=\left(k_{z}+k_{a, z}\right)\left(k_{\mathrm{PC}, z}-k_{b, z}\right)+\eta_{a} \eta_{b}\left(k_{a, z}-k_{\mathrm{PC}, z}\right)\left(k_{z}+k_{b, z}\right) \\
Z_{p}=\left(k_{z} \frac{k_{a}^{2}}{k^{2}}+k_{a, z}\right)\left(k_{\mathrm{PC}, z} \frac{k_{b}^{2}}{k_{\mathrm{PC}}{ }^{2}}-k_{b, z}\right) \\
+\eta_{a} \eta_{b}\left(k_{a, z}-k_{\mathrm{PC}, z} \frac{k_{a}^{2}}{k_{\mathrm{PC}}{ }^{2}}\right)\left(k_{z} \frac{k_{b}^{2}}{k^{2}}+k_{b, z}\right)
\end{gathered}
$$

With the explicit expressions for the $z$ components of the various wave vectors from Section 5, Eqs. (6.4)-(6.13) determine the Fresnel coefficients in terms of $\hat{f}(\omega)$ and $k_{\|^{2}}$ [or of $\hat{f}(\omega)$ and the angle of incidence].

\section{PHASE CONJUGATION}

The incident wave gives rise to an (ordinary) reflected wave, a reflected phase-conjugated wave, and four transmitted waves. It is easy to check from the formulas above that in the limit $\hat{f} \rightarrow 0$ both $T_{a, s}$ and $T_{a, p}$ approach unity and that the other Fresnel coefficients vanish. In this limit the PC is transparent, as it should be. For $\hat{f} \neq 0$ the PC wave appears, but so does the $r$ wave (not to be confused with reflection at a dielectric; this $r$ wave is merely a result of the four-wave mixing). The presence of the $r$ wave already indicates that this device cannot be a perfect $\mathrm{PC}$, even if the $\mathrm{PC}$ wave were the phase-conjugated signal with respect to the incident wave.

Perfect phase conjugation would be achieved if $R_{s}=R_{p}=$ $0, P_{s}=P_{p}=1$, the transmission coefficients were arbitrary, and $\omega_{2}=-\omega_{1}$. With our expressions for the wave vectors and the Fresnel coefficients it is easy to investigate the quality of phase conjugation in a particular situation. Let us first assume that the incident field is on resonance with the PC $\left(\omega_{1}=\bar{\omega}\right)$, which can always be managed by tuning the pump fields. Then we write

$$
\hat{f}(0)=\gamma e^{i \phi}, \quad \gamma>0, \quad \phi \text { real }
$$

so that $\gamma$ measures the strength of the nonlinear interaction. From Eqs. (4.6), (4.7), and (4.13) we then find that

$$
\eta_{a}=-\delta e^{i \phi}, \quad \eta_{b}=\delta e^{-i \phi},
$$

where

$$
\delta=\left\{\begin{aligned}
1 & \text { if } \omega<\bar{\omega} \\
-1 & \text { if } \omega>\bar{\omega}
\end{aligned}\right.
$$

First, we observe that $\left|\eta_{a}\right|=\left|\eta_{b}\right|=1$, and in view of Eqs. (4.1) and (4.2) or (4.8) and (4.9) this means that the $\omega_{1}$ and $\omega_{2}$ waves have the same amplitude, independent of the interaction strength $\gamma$. Second, we notice that $\eta_{a}$ and $\eta_{b}$ are discontinuous if we pass $\omega$ over the resonance $\bar{\omega}$; third, Eq. (7.2) might seem to be in conflict with expressions (4.14) and (4.15). From Eq. (4.7), however, we see that close resonance in fact means that

$$
\frac{\omega_{1}-\left|\omega_{2}\right|}{\omega_{1}} \ll \gamma
$$

or that the relative detuning must be much smaller than the coupling strength. Conversely, the limit $\hat{f} \rightarrow 0$ should read as $\gamma \gg 1-\left|\omega_{2} / \omega_{1}\right|$.

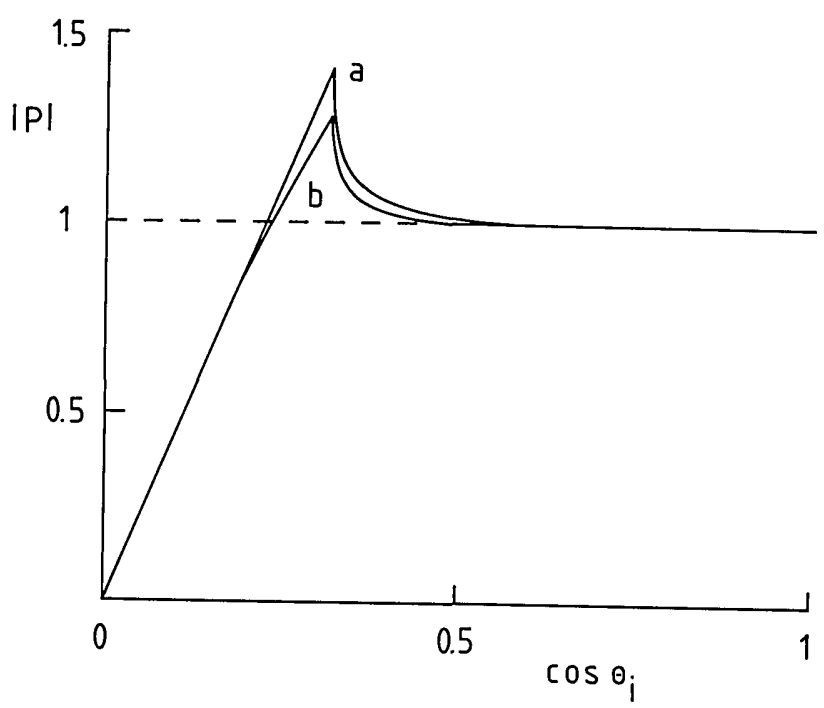

Fig. 2. Reflectivity coefficients $\left|P_{s}\right|$ (curve a) and $\left|P_{p}\right|$ (curve b) for the phase-conjugated wave as a function of the cosine of the angle of incidence. For angles between $0^{\circ}$ and $60^{\circ}$ we have $\left|P_{s}\right| \simeq\left|P_{p}\right| \simeq 1$, which corresponds to perfect phase conjugation. For larger angles, however, the operation of the device is far from perfect, and for parallel incidence the phase-conjugated wave disappears completely. The peaks in the two curves reflect an amplification of the intensity of the phase-conjugated wave with respect to the intensity of the incident wave. The parameters are $\omega_{1}=\bar{\omega}, \gamma=0.1$, and $\phi=0$.

As a second condition we take

$$
\gamma \ll 1,
$$

and subsequently we choose the angle of incidence to be zero $\left(\mathrm{k}_{\|}=0\right)$. Then the Fresnel coefficients simplify considerably, and we obtain

$$
\begin{aligned}
R_{s} & =1 / 2 \delta \gamma \ll 1, \\
P_{s} & =-\delta e^{i \phi}, \\
T_{a, s} & =1, \\
T_{b, s} & =1 / 4 \gamma e^{i \phi} \ll 1 .
\end{aligned}
$$

Equation (7.8) expresses that the amplitude of the incident beam is not affected by the crystal (up to order $\gamma$ ), as could be expected from the fact that for $\gamma \rightarrow 0$ the PC becomes transparent. Furthermore, the ordinary reflected wave and the $b$ waves disappear for $\gamma$ small. Most remarkable is that $\left|P_{s}\right|=1$ in this limit, which implies perfect phase conjugation for a monochromatic wave on resonance and of perpendicular incidence. This feature, which is present for an arbitrarily small nonlinear interaction strength $\gamma$, justifies the name $\mathrm{PC}$ for this device. Figure 2 illustrates the dependence of $\left|P_{s}\right|$ and $\left|P_{p}\right|$ on the angle of incidence.

\section{CONCLUSIONS}

In order to investigate quantitatively the possibilities and limitations of optical phase conjugation, we have worked out the realistic case in which the conjugated wave is generated by four-wave mixing in a nonlinear medium. Since every electromagnetic wave that is incident upon the $\mathrm{PC}$ can be decomposed into plane waves, it is sufficient to evaluate the response of the PC to an external plane wave. We found 
that a PC reflects an ordinary and a phase-conjugated wave back into the vacuum and that the transmitted wave has four components for a half-infinite medium. The wave vectors and Fresnel coefficients were obtained in terms of the incident wave vector and frequency and of the function $\hat{f}(\omega)$, which represents the $\mathrm{PC}$. Reflection and transmission angles for the rays follow from the wave vectors, and the intensities and polarizations are determined by the Fresnel coefficients.

It was shown that the device indeed operates as a PC if the angle of incidence is zero, the wave is on resonance with the $\mathrm{PC}$, and the nonlinear interaction is weak. Conversely, for every other situation the PC conjugates the wave only partially and in addition emits an ordinary reflected wave. In spectroscopic applications, in which spherical waves irradiate the $\mathrm{PC}$, many plane-wave components have a nonzero angle of incidence for which the PC is nonideal. Because it is the interference of all reflected and incident waves that determines, for instance, the lifetime of an atom near the surface, it is inevitable that these imperfections must be taken into account. Moreover, it was pointed out in Section 1 that a perfect phase-conjugating medium should not exist, owing to causality requirements.

\section{ACKNOWLEDGMENTS}

This research was supported by the U.S. Office of Naval Research and by the U.S. Air Force Office of Scientific Research under contract F49620-86-C0009.

T. F. George is also with the Department of Chemistry, State University of New York at Buffalo.

\section{REFERENCES}

1. D. M. Pepper, D. Fekete, and A. Yariv, Appl. Phys. Lett. 33, 41 (1978).

2. C. V. Heer and N. C. Griffen, Opt. Lett. 4, 239 (1979).

3. O. L. Kulikov, N. F. Pilipetskii, A. N. Sudarkin, and V. V. Shkunov, JETP Lett. 31, 345 (1980).

4. B. Ya. Zel'dovich, N. F. Pilipetskii, A. N. Sudarkin, and V. V. Shkunov, Sov. Phys. Dokl. 25, 377 (1980).

5. G. S. Agarwal, A. T. Friberg, and E. Wolf, Opt. Commun. 43, 446 (1982).

6. A. T. Friberg, J. Opt. Soc. Am. 73, 405 (1983).

7. G. S. Agarwal, A. T. Friberg, and E. Wolf, J. Opt. Soc. Am. 73, 529 (1983).

8. F. De Martini, Dipartimento di Fisica, Università di Roma 1, Roma 00185, Italy (personal communication).

9. G. S. Agarwal, Opt. Commun. 42, 205 (1982).

10. R. W. Hellwarth, J. Opt. Soc. Am. 67, 1 (1977).

11. A. Yariv, IEEE J. Quantum Electron. QE-14, 650 (1978).

12. R. A. Fisher, B. R. Suydam, and B. J. Feldman, Phys. Rev. A 23, 3071 (1981).

13. B. R. Suydam and R. A. Fisher, Opt. Eng. 21, 184 (1982).

14. B. R. Suydam, J. Opt. Soc. Am. 73, 539 (1983).

15. M. Nazarathy, A. Hardy, and J. Shamir, J. Opt. Soc. Am. 73, 576 (1983).

16. K. Ujihara, J. Opt. Soc. Am. 73, 610 (1983).

17. M. Ducloy and D. Bloch, Phys. Rev. A 30, 3107 (1984).

18. G. Reiner, P. Meystre, and E. M. Wright, J. Opt. Soc. Am. B 4, 675 (1987).

19. Excellent reviews on the production of phase-conjugated radiation are D. M. Pepper, Opt. Eng. 21, 156 (1982); R. A. Fisher, ed., Optical Phase Conjugation (Academic, New York, 1983); B. Ya. Zel'dovich, N. F. Pilipetskii, and V. V. Shkunov, Principles of Phase Conjugation (Springer-Verlag, Berlin, 1985).

20. A. A. Jacobs, W. R. Tompkin, R. W. Boyd, and E. Wolf, J. Opt. Soc. Am. B 4, 1266 (1987).

21. N. Bloembergen, Nonlinear Optics (Benjamin, London, 1965).

22. Y. R. Shen, The Principles of Nonlinear Optics (Wiley, New York, 1984). 